\title{
Review of the Influencing Factors of Unethical Pro-Organizational Behavior
}

\author{
Caihong Zhang, Xiao Xiao \\ Department of Business Management, Jinan University, Guangzhou, China \\ Email: 13535070201@163.com
}

How to cite this paper: Zhang, C. H., \& Xiao, X. (2020). Review of the Influencing Factors of Unethical Pro-Organizational Behavior. Journal of Human Resource and Sustainability Studies, 8, 35-47. https://doi.org/10.4236/jhrss.2020.81003

Received: January 24, 2020

Accepted: February 24, 2020

Published: February 27, 2020

Copyright $\odot 2020$ by author(s) and Scientific Research Publishing Inc. This work is licensed under the Creative Commons Attribution International License (CC BY 4.0).

http://creativecommons.org/licenses/by/4.0/

\section{(c) (i) Open Access}

\begin{abstract}
The special unethical behavior-unethical pro-organizational behavior has attracted scholars' attention in recent years. It refers to the unethical behaviors which are conducive to the organization that the employees intend to make but violate the social ethical standards. When this concept was first proposed, it has been widely studied by scholars, especially the influencing factors of it. But the content of these researches is rather fragmented and lacks systematic grooming. Based on relevant research in China and abroad, we'll collate and summarize the literature in this field. Firstly, concept, dimensions and measurement of unethical pro-organizational behaviors will be introduced. Then, we'll summarize the influencing factors and mechanisms of it. Finally, based on these, the future research directions in this field will be proposed so that scholars can carry out further research.
\end{abstract}

\section{Keywords}

Unethical Pro-Organizational Behavior, Influencing Factors, Individual Factors, Organizational Factors, Social Factors, Leadership Factors

\section{Introduction}

It is common for employees to abuse their power for personal gain and sacrifice the interests of the organization for their own benefit. This kind of unethical behavior has been paid much attention by researchers (Trevino, Den Nieuwenboer, \& Kishgephart, 2014). Because unethical behavior violates norms and disrupts social order, it will cause different degrees of harm to the society. Therefore, in order to prevent and reduce the occurrence of such behavior, its generation mechanism has been the focus of scholars' research. However, in many cases, employees do such unethical behaviors not for themselves, but for the leadership or organization. For example, employees use false information to exag- 
gerate the company's financial status in order to please the leader, exaggerate the advantages of products to customers in order to increase sales, and conceal product defects in order to protect the interests of the organization. Umphress, Bingham and Mithchell (2010) pointed out that some positive organizational factors such as ethical leadership and organizational support may also be important incentives for unethical behavior. Because these positive factors may increase employees' identification and commitment to the organization, employees may sacrifice social interests for the benefit of the organization, and then perform unethical behavior. This altruistic unethical behavior was defined as unethical pro-organizational behavior, UPB (Umphress, Bingham, \& Mithchell, 2010). Volkswagen's exhaust emission scandal and Toshiba's financial fraud scandal are typical examples of unethical pro-organizational behavior. Because it overturns the understanding of previous studies, the concept of unethical pro-organizational behavior has attracted the attention of scholars since it was put forward. Many scholars have begun to conduct more in-depth studies on this special unethical behavior and explore its mechanism and influencing factors. Some scholars have explored the influence on UPB from the individual factors, such as moral levels (Umphress, Bingham, \& Mitchel, 2010; Umphress \& Bingham, 2011), psychological factors (Ding, 2017), work feelings (Jiang, 2018) and so on. Organizational factors, such as organizational identity (Umphress, Bingham, \& Mitchel, 2010), organizational support (Alexandra, 2012), and organizational ethical climate (Zhang, Jiang, \& Zhao, 2017) have also been proved to positively impact UPB. Besides, leadership has been also shown to affect UPB significantly, such as ethical leadership (Miao et al., 2013), transformational leadership (David, Marc \& Jochen, 2014). In addition, leadership-subordinate exchange (Lin \& Cheng, 2016, 2017) and guanxi (Zhong et al., 2018) has also proven to be an important cause for employees to implement UPB.

Although unethical pro-organizational behavior has caused extensive research by scholars, there are obviously not many researches and relatively little literatures can be consulted. Moreover, although there are relatively more researches on the influencing factors of UPB, the contents are scattered and lack of systematic sorting and integration. Based on it, this paper will comb the existing domestic and foreign literatures in this field, and summarize the concepts, dimensions, measurements, influencing factors and mechanisms of UPB, aiming at providing references for future researchers and proposing future research directions.

\section{Unethical Pro-Organizational Behavior}

\subsection{The Concept of Unethical Pro-Organizational Behavior}

The concept of Unethical Pro-organizational Behavior (UPB) first proposed by Umphress, Bingham and Mitchell in 2010, refers to the unethical behavior that employees intentionally commit in violation of social ethical standards but is beneficial to the organization (Umphress, Bingham, \& Mithchell, 2010). This concept contains two key points: first, UPB is an unethical behavior, which is il- 
legal or unethical for the organization (Jones, 1991). Second, UPB is a kind of behavior that is beneficial to the organization and is actively implemented by employees themselves. However, this concept only emphasizes the benefit of UPB to the organization, but ignores the benefit to the organization members. So Umphress and Bingham came up with a more comprehensive concept in 2011: UPB is the behavior that intentionally implemented by employees to promote the effective functioning of the organization or its members (e.g., leaders) and violate core societal values, mores, laws, or standards of proper conduct (Umphress \& Bingham, 2011). This concept has two core components: UPB is unethical, which means that it violates the hypernorms, or the globally held standards for judging moral behavior from justice, laws or broader social norms. (Donaldson \& Dunfee, 1994). Second, UPB is a unethical behavior that benefits the organization or himself, and the intention behind it is to bring benefits to the members of the organization or both. At the same time, they also give three boundary conditions: firstly, employees may act unethically without specific intent, but UPB is an intentional act that does not include unethical behavior that benefits the organization because of error or unconsciousness; Secondly, employees carry out UPB out of the intention to help the organization. If the behavior's final result brings trouble to the organization, it does not belong to UPB. Lastly, UPB emphasizes that behaviors are mainly for the purpose of improving the interests of the organization, unethical behaviors conducted primarily with the intention of benefiting the self alone, and not the organization or its members, would not be considered UPB.

\subsection{The Differences between UPB and Other Similar Concepts}

\section{1) Necessary Evils}

Molinsky and Margolis (2005) suggest that employees sometimes act for the benefit of individuals, organizations, or society, but may hurt others during the course of the action. They call these behaviors "necessary evils" and define it as "work-related tasks in which an individual must, as a part of his or her job, perform an act that causes emotional or physical harm to another human being in the service of achieving some perceived greater good." (Molinsky \& Margolis, 2005). For example, managers tell subordinates bad news about work, medical staff provide painful treatment to patients, teachers give negative feedback to students, and so on. Like UPB, necessary evils can benefit the organization, but also be beneficial to the society, which is beyond the scope of UPB. And necessary evils include not only ethical behavior, but also ethical behavior.

2) Deviance

Warren (2003) define deviance from two dimensions: first, whether the behavior conforms to or violates the hypernorms or social moral standards; Second, whether the behavior conforms to or deviates from the norms within the organization. Deviance may sometimes conform to the norms within the organization, but violate the social moral standards (Warren, 2003). The difference between 
UPB and deviance lies in: first, the victims of UPB are usually outside the organization, but the victims of deviance may be employees or organizations; second, deviance is not only unethical behavior, but all of UPB are unethical behaviors.

3) Organization Misbehavior

Vardi and Weitz (2004) proposed a new type of unethical behavior based on the intention of deviance, that is, organization misbehavior. Organization misbehavior includes three aspects: first, acts intended to benefit the self; second, acts intended to harm others or the organization; and third, acts intended to benefit the organization (Vardi \& Weitz, 2004). There are two differences between UPB and organization misbehavior: firstly, UPB is based on the theory of social exchange, but organization misbehavior is not. Second, organization behavior is either a violation of social behavior standards or a violation of organizational norms, but regardless of whether or not the behavior meets organizational expectations, UPB only focuses on behaviors that violate social behavior standards.

4) Pro-social Rule Breaking

Pro-social rule breaking refers to employees' behaviors that intentionally violate formal organizational policies, regulations or prohibitions for the sake of the organization or stakeholders (Morrison, 2006). The similarities between UPB and pro-social rule breaking are: first, the motivation for both is for the organization or its employees. Second, both are intentional acts that are performed consciously. Third, both are behaviors outside the scope of the employee's responsibility. The differences between these two behaviors are: firstly, UPB violates social behavior standards, while pro-social rule-breaking violates organizational norms; secondly, UPB will cause harm to society, but pro-social rule-breaking does not.

\subsection{The Dimensions and Measurements of UPB}

As for the dimensions of UPB, in the concept proposed by Umphress, Bingham and Mitchel (2010), three scholars believe that UPB is only a one-dimensional concept of pro-organizational behavior, that is, UPB can be an unethical pro-organization, an unethical pro-member, or an unethical pro-organization and pro-member behavior. However, Xia (2014) proposed that UPB is a two-dimension concept, including altruism and pro-organization. The dimension of altruism is the unethical behavior that is beneficial to the members of the organization without harming the interests of the organization, while the dimension of pro-organization is the unethical behavior that is beneficial to the organization.

Regarding the measurement scale of UPB, the earliest is a one-dimensional structure scale with six items developed by Umphress, Bingham and Mitchel (2010). The scale uses a seven-point scale from 1 (very disagree) to 7 (strongly agree) to distinguish employees' willingness to engage in UPB. The items include, for example, "If it would help my organization, I would misrepresent the truth to make my organization look good". This measurement scale has been 
proved to have good reliability. Later, in order to study the UPB frequency of employees in fast-food chain companies, Matherne and Litchfield (2012) also developed a "UPB 5-item scale", which uses a seven-point scale from 1 (never) to 7 (always) to distinguish the frequency of UPB. Items include, for example, "I am willing to forge documents to protect my company". The scale also has good reliability. However, it has not been verified by researchers on a large scale, so it has not been widely used by scholars.

\section{The Influencing Factors of UPB}

Studies have shown that UPB is affected by a variety of factors, and factors at different levels such as the individual, organization, leadership, and society levels will have an important impact on it. In order to explore which factors will affect UPB and how to influence them, this article summarizes the relevant literature at home and abroad, and lists the following four levels of factors affecting UPB and their mechanisms of action.

\subsection{Individual Level}

As the subject of UPB, individuals' behavioral intentions will be affected by their own characteristics. Individual psychological factors mainly exist as mediators and moderators in the study of UPB. For example, the positive reciprocity beliefs held by individuals and the moral development of individuals (Umphress, Bingham, \& Mitchel, 2010; Umphress \& Bingham, 2011), personal disposition toward ethical/unethical behavior (David, Marc \& Jochen, 2014), moral identity (Wu et al., 2016), psychological ownership (Ding, 2017), moral disengagement (Zhao \& Zhou, 2017) and other variables. Besides, the level of ego orientation of employees through the impact of its moral disengagement level and the influence UPB (Liu, 2018). In addition, the higher the risk of ostracism, the easier it is for employees to engage in UPB to gain recognition and approval from the organization, so as to solve the crisis of ostracism (Thau et al., 2015; Zhang, 2016). And the high performance expectation (Chen \& Liang, 2017), the high performance pressure (Li et al., 2018) and job insecurity (Jiang, 2018) felt by employees have also been proved to induce employees to implement UPB.

\subsection{Organizational Level}

As the main object of UPB, organization must have an important influence on it. Empirical research has shown that there is a positive correlation between organizational identity and UPB, and individuals' positive reciprocity beliefs have a moderating effect on this relationship (Umphress, Bingham, \& Mitchel, 2010). Alexandra (2012) also found that organizational support has a significant positive promotion effect on UPB, organizational support can influence employees' moral judgment, thus prompting them to conduct UPB. Moreover, moral leadership moderates the impact of organizational support on employees' moral judgments. In addition, Organizational commitment is also an important factor 
leading to UPB. Matherne and Litchfield (2012) found that employees with high organizational emotional commitment are more likely to make UPB for the benefit of the organization, and this relationship is affected by personal moral identity. Herchen et al. (2012) proposed that organizational culture will promote the emergence of employees' UPB. Organizational culture can transmit the signals of organizational normative behavior to employees through organizational expectations and values. Through immoral socialization and loose ethical regulations, employees misestimate the organization's acceptance of unethical behaviors, which triggers a series of unethical behaviors. Besides, organizational ethical climate also has been proved to have a significant impact on UPB (Zhang, Jiang, \& Zhao, 2017). Instrumental ethical climate and caring ethical climate had a positive effect on UPB, while principle ethical climate has a negative effect on UPB. In addition, high commitment human resource management practices have been proved to have a positive impact on UPB, and in this relationship, organizational support plays a fully mediating role, while moral identity plays a negative regulating role (Luo \& $\mathrm{Xu}, 2017$ ).

\subsection{Social Level}

There are relatively few studies on the factors that affect UPB at the social level, mainly focusing on the application of social exchange theory. In a positive social exchange relationship, employees are likely to repay the favor given by the organization through various behaviors, even unethical behaviors (Blau, 1964). Research by Umphress and Bingham (2011) confirms it. They explored the impact of social exchange and organizational identity on UPB. Both social exchange and organizational identity have a positive impact on UPB (Umphress \& Bingham, 2011). In addition, Lin and Cheng $(2016,2017)$ showed through empirical research that leadership-subordinate exchange and differential leadership have a significant positive impact on UPB.

Zhong et al. (2018) explored the relationship between superior-subordinate Guanxi and UPB. The study found that these two variables were significantly positively related, and that organizational identity played a mediating role between the relationship.

\subsection{Leadership Level}

As the role model of employees in the organization, leaders will have an important influence on employees' ethical cognition and behavior (David, Marc, \& Jochen, 2014; Kalshoven et al., 2016). At the level of leadership, there are relatively more studies on the influence of leadership style on UPB. Different leadership styles exert different influences on UPB through different influence mechanisms.

There is an inverted u-shaped relationship between ethical leadership and employees' UPB. In this relationship, organizational identity plays a mediating role, identification with supervisor (Miao et al., 2013) and chronic regulatory focus (Li, 2016). However, Kalshoven et al. (2016) proposed that there is no in- 
verted u-shaped relationship between ethical leadership and employees' UPB, but a positive correlation, possibly because of sample differences.

Transformational leadership promotes employees' UPB by improving their organization identification, employees' personal disposition toward ethical and unethical behavior and supervisor's organizational embodiment play a moderating role, respectively (David, Marc, \& Jochen, 2014; Wang \& Ying, 2018). Graham et al. (2015) studied the influence of leadership style on employees' UPB under the regulation of farming, and found that inspirational and charismatic transformational leaders stimulated higher levels of UPB, and followers' promotion regulatory focus moderated this relationship such that the effect held for followers with low promotion focus, but not for individuals with high promotion focus.

Among paternalistic leadership, Zhang, Zhang and Zhao (2017) found that authoritarian leadership positively affect UPB, and the relationship between moral leadership and UPB is an inverted U shape, authoritarian leadership, benevolent leadership and moral leadership have a significant interaction on UPBs. Authoritarian leadership has a stronger positive impact on the UPB of highly traditional individuals. The inverted U-shaped curve relationship between the moral leadership and UPB is also affected by traditional adjustment. Besides, research has also shown that authoritarian leadership has a significant predictive effect on UPB, and the dual interaction of benevolent and authoritarian leadership, moral leadership and authoritative leadership positively affect UPB. LMX plays a moderating role between paternalistic leadership and UPB (Li et al., 2019).

Regarding the impact of differential leadership on UPB, Lin and Cheng (2016) and Zhang (2016) discussed the formation mechanism of insiders or outsiders' UPB from the perspective of a single insider or outsider, respectively. Result shows that differential leadership has a positive impact on both insiders' and outsiders' UPB. Later, Lin and Cheng (2017) explored the impact mechanism of differential leadership on employees' UPB from the perspectives of insiders and outsiders, and found that differential leadership has a positive effect on insiders' and outsiders' UPB, however the difference of effect degree between them is not significant.

As for the influence of servant leadership on employees' UPB, Wu et al. (2017) found an inverted u-shaped relationship between servant leadership and UPB, and moral identity internalization moderated this inverted U-shaped relationship. When the employees' moral identity internalization level is low, the relationship between the servant leadership and the employees' UPB is inverted u-shaped, while employees' moral identity internalization level is high, the relationship does not exist. The reason may be that individuals with higher moral identity internalization level are more likely to follow moral rules than individuals with lower moral identity internalization level (Mulder \& Aquino, 2013). Besides, it may be that individuals with moral identity symbolization do not truly identify with moral norms from their heart (Aquino \& Reed, 2002), so moral identity symbolization does not moderate the relationship between servant lea- 
dership and UPB.

Finally, empowering leadership has also been proved to have a significant positive effect on UPB of employees, and psychological empowerment and LMX played a chain mediating role between them (Xu, Wang, \& Fan, 2018). Moreover, the mediating effect of LMX was higher than psychological empowerment.

\section{Summary and Implication}

\subsection{Summary}

From the above review, we can find that in the current research, the influencing factors of UPB are mainly concentrated at four levels: individual, organization, society and leadership, and different factors have different effects on UPB through different influence mechanisms, as shown in Figure 1.

It can be seen that although in the previous research, some positive factors such as organizational support, LMX and ethical leadership can strengthen the employees' organizational identity, increase the employees' enthusiasm, and bring benefits to the organization, but in fact at the same time, these will also encourage employees to make unethical behaviors for organizations. Although UPB can benefit organizations in the short term, it is not conducive to the development of organizations in the long run. Therefore, organization leaders must be vigilant and strictly regulate subordinates' behaviors, so as to create a positive organizational atmosphere, and avoid inducing employees to conduct UPB.

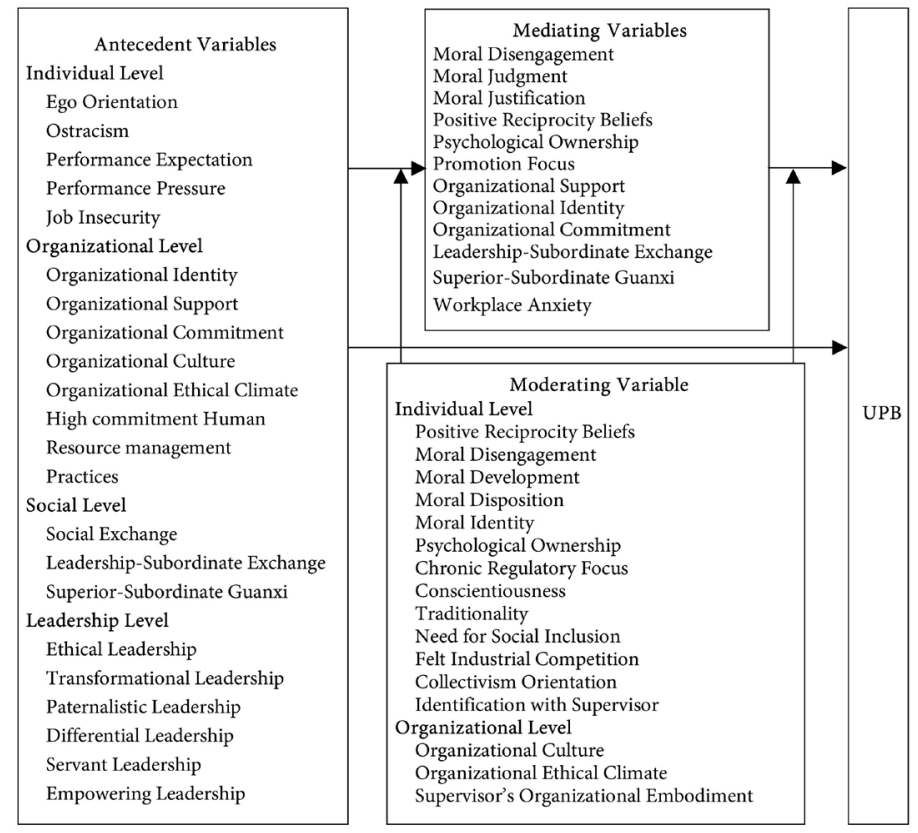

Figure 1. The influencing factors of UPB.

\subsection{Theoretical Significance}

This paper has summarized the influencing factors of UPB from different levels, 
the theoretical significance is as follow:

- We have summarized the relevant empirical researches on the influencing factors of UPB from four aspects: individual level, organizational level, social level and leadership level, and clarified the reasons and purposes that can induce employees to implement UPB, so that scholars can have a clearer understanding of UPB. Therefore, the content system of UPB research has been enriched.

- Based on the analysis of the existing research limitations, we look forward to the future research directions from five aspects to provide the follow-up scholars with research directions and strive to further enrich the theoretical research of UPB.

\subsection{Practical Significance}

Although UPB can benefit the organization in the short term, in the long run it will inevitably harm the interests of the organization and weaken the long-term competitiveness of the enterprise due to its violation of ethics. Therefore, the organization must take measures to restrain employees from implementing UPB. This paper has the following three management implications:

- Organizations should create a positive ethical atmosphere and an organizational culture that advocates ethics. Self-interested ethical climate may induce employees to implement UPB, so organization should strengthen the construction of a rule-based ethical climate, further formulate relevant laws and regulations, codes of conduct such as business ethics and professional ethics, and require employees to implement them, so as to induce employees to repay the organization for helping others in a correct and ethical way.

- Organization should improve the moral level of leaders, and leaders also need to improve their values continuously. Transformational leadership, servant leadership, ethical leadership, etc. may induce employees to implement UPB, which have certain negative effects, leaders should lead by example. When employees are found to benefit the organization through unethical means, leaders should not ignore, acquiesce or even support them. Instead, they should timely and effectively control UPB, add ethical considerations to performance evaluation and establish a sound mechanism of moral rewards and punishments.

- The organization should pay attention to employees' moral cognition and improve their moral level. Employees with low moral standards are more likely to engage in UPB, so organization should concern the moral level of employees and organize relevant training to change the unethical moral cognition of employees from the source, guide employees to help organization by acting ethically, instead of actions that harm organization and themselves.

\subsection{Limitations and Future Prospects}

After reviewing the relevant literature on UPB's four levels of influencing factors, 
we believe that there are still some limitations and some problems need to be further discussed. Therefore, we put forward the following unresearched directions.

- Unlike the West, China has attached great importance to social relations since ancient times. People are divided into outsiders and insiders according to the degree of intimacy, and different moral standards may be applied to family members and outsiders. The Chinese pursue moral particularism, and moral standards vary with time, place and person. However, UPB was put forward by western scholars based on the western context. Therefore, future research needs to put forward a localized concept of UPB based on Chinese culture, instead of simply following the western concept. In addition, its conceptual structure and measurement standards need to be clarified, and the influence of leadership style in Chinese context, such as paternalistic leadership and differential leadership, on employees' UPB can be further studied, so as to better avoid employees' UPB in Chinese context.

- In the current research, there are few studies on factors at the individual level and social level as antecedent variables of UPB, and the social level only involves the concept of social exchange. There are many other individuals and social factors that are closely related to UPB. Individual factors such as control points, self-concepts, and interpersonal orientations, and social factors such as social networks and social norms are closely related to UPB. Future research can start from these factors and analyze the connection between them and UPB in all aspects.

- The research about the impact of ethical leadership on employee UPB may be the reason for the research sample, and no conclusion has been reached at present. Future researches can further explore the relationship between ethical leadership and UPB, or study the influence of ethical leadership on UPB in different situations, and draw more accurate and universal conclusions.

- Although there are relatively more studies on the influence of leadership factors on UPB, other leadership factors may also have a certain impact on employees' UPB, such as leadership expectations and leadership characteristics, which also are directions worthy of research in the future. In addition, future research can also explore the transformation between different leadership styles, as well as what leadership trait styles can inhibit the UPB of employees in different organizational environments.

- This paper does not review the outcome variables of UPB. Although there are few researches on the results of UPB, there is still a lack of collation and summary of them. Moreover, the results of UPB, especially the positive results that can induce employees to carry out such behavior, still deserve further discussion in the future, so as to find out more purposes for employees to carry out such behavior and stop this behavior.

\section{Conflicts of Interest}

The authors declare no conflicts of interest regarding the publication of this paper. 


\section{References}

Alexandra, I. (2012). Unethical Pro-Organizational Behaviors: Antecedents and Boundary Conditions. Tampa, FL: University of South Florida.

Aquino, K., \& Reed, A. I. (2002). The Self-Importance of Moral Identity. Journal of Personality and Social Psychology, 83, 1423-1440. https://doi.org/10.1037/0022-3514.83.6.1423

Blau, P. M. (1964). Exchange and Power in Social Life. New York: John Wiley \& Sons.

Chen, M., \& Liang, J. (2017). High Performance Expectation and Unethical Pro-Organizational Behavior: Social Cognitive Perspective. Acta Psychologica Sinica, 49, 94-105. https://doi.org/10.3724/SP.J.1041.2017.00094

David, E., Marc, S., \& Jochen, G. (2014). Transformational Leadership and Follower's Unethical Behavior for the Benefit of the Company: A Two-Study Investigation. Journal of Business Ethics, 120, 81-93. https://doi.org/10.1007/s10551-013-1644-Z

Donaldson, T., \& Dunfee, T. W. (1994). Toward a Unified Conception of Business Ethics: Integrative Social Contracts Theory. Academy of Management Review, 19, 252-284. https://doi.org/10.5465/amr.1994.9410210749

Graham, K. A., Ziegert, J. C., \& Capitano, J. (2015). The Effect of Leadership Style, Framing, and Promotion Regulatory Focus on Unethical Pro-Organizational Behavior. Journal of Business Ethics, 126, 423-436. https://doi.org/10.1007/s10551-013-1952-3

Jiang, X. Y. (2018). The Formation Mechanism of Unethical Pro-Organizational Behavior: A Test of an Interactive Model. Henan: Henan University.

Kalshoven, K., Van, Dijk, H., \& Boon, C. (2016). Why and When Does Ethical Leadership Evoke Unethical Follower Behavior? Journal of Managerial Psychology, 31, 500-515. https://doi.org/10.1108/JMP-10-2014-0314

Li, G. Q. (2016). Ethical Leadership, Organizational Identification and Employees' Unethical Pro-Organizational Behavior: The Moderate Effect of Chronic Regulatory Focus. Science of Science \& Management of S \& T, 37, 125-135.

Li, Z. C., Wang, Z., Zhu, Z. B., \& Zhan, X. J. (2018). Performance Pressure and Unethical Pro-Organizational Behavior: Based on Cognitive Appraisal Theory of Emotion. Chinese Journal of Management, 15, 358-365.

Li, Z. Y., Li, X. Q., Xu, H. C., \& Wu, M. Z. (2019). The Effect of Paternalistic Leadership on Unethical Pro-Organizational Behaviors: The Moderating Role of Leader-Member Exchange. Psychological Research, 12340-348.

Lin, Y. H., \& Cheng, K. (2016). Leader-Member Exchange and Employees' Unethical Pro-Organizational Behavior: A Differential Mode Perspective. Journal of Management Science, 29, 57-70.

Lin, Y. H., \& Cheng, K. (2017). Differential Leadership and Employees' Unethical Pro-Organizational Behavior: A Perspective of Insider and Outsider. Journal of Management Science, 30, 35-50.

Liu, J. M. (2018). Study on the Impact of Moral Disengagement and Organizational Ethics Climate on the Relationship of Employee's Ego Orientation and Unethical Pro-Organizational Behavior. Hangzhou: Zhejiang Gongshang University.

Luo, F., \& Xu, R. H. (2017). The Effect of High Commitment Human Resource Management Practices on Unethical Pro-Organizational Behavior: The Mediating Role of Perceived Organizational Support and the Moderating Role of Moral Identity. Human Resources Development of China, No. 10, 28-38.

Matherne, I. C. F., \& Litchfield, S. R. (2012). Investigating the Relationship between Af- 
fective Commitment and Unethical Pro-Organizational Behaviors: The Role of Moral Identity. Journal of Leadership Accountability \& Ethics, 9, 35-46.

Miao, Q., Newman, A., Yu, J., \& Xu, L. (2013). The Relationship between Ethical Leadership and Unethical Pro-Organizational Behavior: Linear or Curvilinear Effects? Journal of Business Ethics, 116, 641-653. https://doi.org/10.1007/s10551-012-1504-2

Molinsky, A., \& Margolis, J. D. (2005). Necessary Evils and Interpersonal Sensitivity in Organizations. The Academy of Management Review, 30, 245-268. https://doi.org/10.5465/amr.2005.16387884

Morrison, E. W. (2006). Doing the Job Well: An Investigation of Pro-Social Rule Breaking. Journal of Management, 32, 5-28. https://doi.org/10.1177/0149206305277790

Mulder, L. B., \& Aquino, K. (2013). The Role of Moral Identity in the Aftermath of Dishonesty. Organizational Behavior and Human Decision Processes, 121, 219-230. https://doi.org/10.1016/j.obhdp.2013.03.005

Thau, S., Derfler-Rozin, R., Pitesa, M., Mitchell, M. S., \& Pillutla, M. M. (2015). Unethical for the Sake of the Group: Risk of Social Exclusion and Pro-Group Unethical Behavior. Journal of Applied Psychology, 100, 98-113. https://doi.org/10.1037/a0036708

Trevino, L. K., Den Nieuwenboer, N. A., \& Kishgephart, J. J. (2014). (Un)Ethical Behavior in Organizations. Annual Review of Psychology, 65, 635-660. https://doi.org/10.1146/annurev-psych-113011-143745

Umphress, E. E., \& Bingham, J. B. (2011). When Employees Do Bad Things for Good Reasons: Examining Unethical Pro-Organizational Behaviors. Organization Science, 22, 621-640. https://doi.org/10.1287/orsc.1100.0559

Umphress, E. E., Bingham, J. B., \& Mitchell, M. S. (2010). Unethical Behavior in the Name of the Company: The Moderating Effect of Organizational Identification and Positive Reciprocity Beliefs on Unethical Pro-Organizational Behavior. Journal of Applied Psychology, 95, 769-780. https://doi.org/10.1037/a0019214

Vardi, Y., \& Weitz, E. (2004). Misbehavior in Organizations: Theory, Research, and Management. Mahwah, NJ: Lawrence Erlbaum Associates. https://doi.org/10.4324/9781410609052

Wang, X. C., \& Ying, Y. (2018). How Does Transformational Leadership Influence Employees' Unethical Pro-Organizational Behavior?-A Moderated Mediating Model. Collected Essays on Finance and Economics, No. 3, 97-104.

Warren, D. E. (2003). Constructive and Destructive Deviance in Organizations. The Academy of Management Review, 28, 622-632. https://doi.org/10.2307/30040751

Wu, M. Z., Shao, X. L., Sun, X. L., \& Li, N. (2017). Research on the Relationship among Servant Leadership, Moral Identity and Unethical Pro-Organization Behaviors. Chinese Journal of Applied Psychology, 23, 152-161.

Wu, M. Z., Shen, B., \& Sun, X. L. (2016). The Relationship between Organizational Commitment and Unethical Pro-Organizational Behaviors: The Moderating Role of Moral Identity. Journal of Psychological Science, 39, 392-398.

Xia, F. B. (2014). A Integrated Research of Employee Unethical Pro-Organizational Behavior's Antecedents and Consequences. Ph.D. Thesis, Dalian: Dongbei University of Finance and Economics.

Xu, L., Wang, J., \& Fan, C. H. (2018). The Study of the Impact of Empowering leadership on Unethical Pro-Organizational Behavior of Employees: A Chain Mediating Model. Science of Science and Management of S.\&.T., 39, 109-121.

Zhang, G. P. (2016). The Influencing Mechanism of Workplace Ostracism on Unethical 
Pro-Organization Behavior. Journal of Management Science, 29, 104-114.

Zhang, Y. J., Jiang X. Y., \& Zhao, G. X. (2017). The Relationship between Ethical Climate and Unethical Pro-Organizational Behavior: Moral Justification as a Mediator. Journal of Psychological Science, 40, 1189-1194.

Zhang, Y. J., Zhang, P. C., \& Zhao, J. (2017). The Relationship between Paternalistic Leadership and Unethical Pro-Organizational Behavior: Traditionality as a Moderator. Nankai Business Review, 20, 169-179.

Zhao, H. D., \& Zhou, J. (2017). Corporate Hypocrisy, Moral Disengagement and Unethical Pro-Organizational Behavior: Moderated Mediating Effect. Foreign Economics \& Management, 39, 15-28.

Zhong, X., Wang, T., Luo, H. Y., \& Song, T. B. (2018). The Effect of Supervisor-Subordinate Guanxi on Unethical Pro-Organizational Behavior: The Role of Organizational Identification and Self-Sacrificial Leadership. Science of Science and Management of S.\&.T., 39, 122-135. 